International Journal of Physical Research, 7(1) (2019) 3-6
International Journal of Physical Research
SPC
Website: www.sciencepubco.com/index.php/IJPR
Research paper

\title{
Theoretical investigation of acoustic wave velocity of aluminum phosphide under pressure
}

\author{
Salah Daoud ${ }^{1}$, Abdelhakim Latreche ${ }^{2}$, Pawan Kumar Saini ${ }^{3}$ \\ ${ }^{1}$ Laboratory of Materials and Electronic Systems (LMSE), Mohamed Elbachir El Ibrahimi, \\ Bordj Bou Arreridj University, 34000 Bordj Bou Arreridj, Algeria \\ ${ }^{2}$ Département des Sciences de la matière, Université Mohamed Elbachir El Ibrahimi de Bordj Bou Arreridj, \\ Bordj Bou Arreridj, 34000, Algérie \\ ${ }^{3}$ Department of Physics, Government College, Hansi, Haryana, 125033, India \\ *Corresponding author E-mail: salah_daoud07@yahoo.fr
}

\begin{abstract}
The bulk and surface acoustic wave velocities of Aluminum phosphide (AlP) semiconducting material under pressure up to 9.5 GPa were studied. The structural parameters and the elastic constants used in this work are taken from our previous paper published in J. Optoelectron. Adv. M. 16, 207 (2014). The results obtained at zero-pressure are analyzed and compared with other data of the literature. In addition, the acoustic Grüneisen parameter and the Vickers and Knoop microhardness are predicted and analyzed in detail. Our calculated results are in good agreement with the experimental and other theoretical data of literature.
\end{abstract}

Keywords: Alp Semiconductor; Bulk and Surface Acoustic Wave Velocities; Newton-Raphson's Iterative Method; High Pressure.

\section{Introduction}

The binary compounds of III-V group's and their ternary alloys have been the subject of intense investigation in recent theoretical and experimental studies [1-7] due to their important physical properties such as: large bulk modulus, good hardness, and wide band-gap [1]. Among the binary compounds: AlP material, which crystallizes in cubic zincblende phase. It is an indirect wide band gap semiconductor $(\mathrm{Eg}=2.48 \mathrm{eV})$, it has enormous technological importance in the industry of the infrared photo-detectors and light emitting diodes [1]. Recently, Aouadi et al. [2] studied some important physical properties of AlP material in both B3 and nickel-arsenide (B8) phases using PP-PW approach. Lakel et al. [3] preceded a systematic study of the effect of the pressure on the structural parameters, mechanical moduli and lattice dynamics properties of AlP material in both B3 and B8 phases.

Based on the idea, that the physical properties of elements and binary compounds can be improved artificially by adding some other constitutions to elaborate ternary alloys, Ameri et al. [4] have reported FP-LMTO calculations for equilibrium structure and the electronic properties of AlP and InP binary compounds and the effect of $\mathrm{Al}$ concentration on some physical properties of $\mathrm{Al}_{\mathrm{x}} \mathrm{In}_{1-\mathrm{x}} \mathrm{P}$ ternary alloys combining AlP and InP binary compounds. The effect of the high pressure on the mechanical properties of BP, AlP, GaP and InP binary compounds were investigated by Bouhemadou et al. [5] using FP-APW + lo method.

Ehsanfar et al. [6] have performed a systematic investigation of the structural parameters, elastic constants, sound velocities, and some other physical properties of four III-phosphide binary compounds (BP, AlP, GaP and InP) in B3 phase using the DFT and the DFPT methods implemented in the Quantum ESPRESSO package. They also investigated the phonon spectrum of these compounds using the PHON program. They found that the frequency of the central optical phonon modes for AlP compound is around $427 \mathrm{~cm}^{-1}$, which is localized between $337 \mathrm{~cm}^{-1}$ and $783 \mathrm{~cm}^{-1}$ of GaP and BP, respectively [6].

In our previous work [7], the elastic and electronic properties of AlP material were investigated. In order to extend our research on this compound (AIP), we have calculated some other important parameters which are lacking in literature.

In the present work, as a part of this series of compounds, a bulk and surface-acoustic wave speeds up to 9.5 GPa are predicted. Our results obtained at equilibrium are analyzed and compared with the previous calculations and available experimental results of the literature.

\section{Theory, results and discussion}

\section{1. Elastic wave velocities for some specific crystallographic directions}

Sound wave propagates through a solid by three acoustical modes, one of them is longitudinal and others two are transverse in nature. Hence, there are three types of velocities exits, one is the longitudinal wave velocity $\left(\mathrm{V}_{\mathrm{p}}\right)$ and others two are the shear waves velocities $\left(\mathrm{V}_{\mathrm{s} 1}, \mathrm{~V}_{\mathrm{s} 2}\right)$. These three types of velocities depend on the direction of the propagation of the wave in the crystal [8]. The expressions for 
direction dependent ultrasonic velocities in cubic crystals can be described as function of the elastic constants $\mathrm{C}_{\mathrm{ij}}$ and the density $\rho$ as follows [8]. The different relationships used here are detailed elsewhere [9]. Our obtained values of the longitudinal-wave mode speed $\left(\mathrm{V}_{\mathrm{p}}\right)$ and transverse-wave mode speed $\mathrm{V}_{\mathrm{s} 1}, \mathrm{~V}_{\mathrm{s} 2}$ in AlP material propagating in [100], [110] and [111] directions for different values of applied pressure up 9.5 GPa are given in Table 1, and compared with available data of the literature [6], [9], [10].

Table 1: Sound Velocities in (m/s) for Major Directions in AlP Up to 9.5 GPa. ${ }^{\mathrm{a}}$ Longitudinal Waves, ${ }^{\mathrm{b}}$ Shear Waves

\begin{tabular}{|c|c|c|c|c|c|}
\hline \multirow{2}{*}{ Propagation (Direction) } & \multirow{2}{*}{ Plane of Polarization } & \multicolumn{4}{|c|}{ At equilibrium $(\mathrm{P}=0 \mathrm{GPa})$} \\
\hline & & This work & Ref. [6] & Ref. [9] & Ref. [10] \\
\hline \multirow{2}{*}{ [100] } & {$[100]^{\mathrm{a}}$} & 7430 & 7710 & 7285 & 7970 \\
\hline & $(100)^{b}$ & 5093 & 4550 & 5172 & 5090 \\
\hline \multirow[t]{2}{*}{ [110] } & $(001)^{b}$ & 5093 & 4550 & 5172 & 5090 \\
\hline & $(110)^{b}$ & 3649 & 4230 & 5213 & 4260 \\
\hline \multirow{2}{*}{ [111] } & {$[111]^{\mathrm{a}}$} & 8487 & 7950 & 8404 & 8590 \\
\hline & $(111)^{\mathrm{b}}$ & 4186 & 4340 & 4240 & 4560 \\
\hline
\end{tabular}

\begin{tabular}{|c|c|c|c|c|c|c|}
\hline \multirow{2}{*}{ Propagation (Direction) } & \multirow{2}{*}{ Plane of Polarization } & \multicolumn{4}{|c|}{ Pressure $(\mathrm{GPa})$} & \multirow[b]{2}{*}{9.50} \\
\hline & & 1.46 & 3.17 & 4.87 & 6.50 & \\
\hline \multirow{2}{*}{ [100] } & {$[100]^{\mathrm{a}}$} & 6730 & 6395 & 6187 & 6053 & 5892 \\
\hline & $(100)^{b}$ & 4560 & 4274 & 4077 & 3933 & 3709 \\
\hline \multirow{3}{*}{ [110] } & {$[100]^{\mathrm{a}}$} & 7458 & 7083 & 6847 & 6691 & 6480 \\
\hline & $(001)^{\mathrm{b}}$ & 4560 & 4274 & 4077 & 3933 & 3709 \\
\hline & $(110)^{b}$ & 3235 & 3000 & 2833 & 2833 & 2546 \\
\hline \multirow{2}{*}{ [111] } & {$[111]^{\mathrm{a}}$} & 7685 & 7298 & 7053 & 6891 & 6664 \\
\hline & $(111)^{b}$ & 3730 & 3477 & 3300 & 3170 & 2985 \\
\hline
\end{tabular}

As seen from this table, $\mathrm{V}_{\mathrm{p}}$ are fastest along [111] direction and the shear waves are slowest along [100] direction. On comparing results of the sound velocities for major directions with those obtained by Ehsanfar et al. [6], Ma et al. [9] and Adachi [10], one can notice a good agreement, except for $\mathrm{V}_{\mathrm{s} 1}$ in the [110] direction, a slightly difference was observed. However, to the best of the authors' knowledge, no theoretical or experimental data of the sound velocities for major directions at high-pressure have appeared anywhere in literature to compare our results. Therefore, this work can be used as a reference for any further experimental or theoretical study of this compound.

\section{2. Elastic wave velocities of the aggregate material}

For the aggregate materials (polycrystalline compounds), the Debye average velocity $\mathrm{vm}$ is usually used to study the ultrasonic velocities wave propagation; this quantity is given by the following expression [11], [12]

$v_{m}=\left[(1 / 3)\left(\left(2 / v_{i}{ }^{3}\right)+\left(1 / v_{1}{ }^{3}\right)\right)\right]^{-1 / 3}$

Where: $\mathrm{vl}_{\mathrm{l}}$ and $\mathrm{v}_{\mathrm{t}}$ are the longitudinal and transverse elastic wave velocities respectively. They are determined by using the following expressions [11]: $\mathrm{vl}_{\mathrm{l}}=((3 \mathrm{~B}+4 \mathrm{G}) / 3 \mathrm{~g})^{1 / 2}$, and $\mathrm{vt}_{\mathrm{t}}=(\mathrm{G} / \mathrm{g})^{1 / 2}$, where: $\mathrm{B}$ is the bulk modulus, $\mathrm{G}$ is the shear modulus, and $\mathrm{g}$ is the density.

The computed values of $\mathrm{v}_{\mathrm{l}}, \mathrm{v}_{\mathrm{t}}$ and $\mathrm{v}_{\mathrm{m}}$ of AlP at zero-pressure are: $7994 \mathrm{~m} / \mathrm{s}, 4455 \mathrm{~m} / \mathrm{s}$ and $4960 \mathrm{~m} / \mathrm{s}$, respectively. They are specified in Table 2, and compared with available data of the literature [5], [9].

Our obtained results are in good agreement with the theoretical ones reported by Bouhemadou et al. [5] and Ma et al. [9] and a small deviation about $1.1 \%$. was found between our value $(4960 \mathrm{~m} / \mathrm{s})$ of $\mathrm{v}_{\mathrm{m}}$ and the value $(5015 \mathrm{~m} / \mathrm{s})$ reported by Ma et al. [9].

Table 2: Sound Velocities (in m/s) of AlP Compound at Zero-Pressure, in Comparison with Other Data of the Literature [5], [9]

\begin{tabular}{llll}
\hline Parameter & $\mathrm{V}_{\mathrm{l}}$ & $\mathrm{V}_{\mathrm{S}}$ & \\
\hline This work & 7994 & 4455 & $\mathrm{~V}_{\mathrm{m}}$ \\
Ref. [5] & 8426 & 4981 & 4960 \\
Ref. [9] & 7873 & 4514 & 5518 \\
\hline
\end{tabular}

In Figure 1, the transverse, longitudinal, and average sound velocities are plotted against pressure. It is found that all the sound velocities decrease monotonically with increasing pressure.



Fig. 1: Longitudinal, Transverse and Average Sound Velocities of AlP Semiconductor versus Pressure. 
By best fitting the longitudinal, transverse, and average acoustic wave velocities, the following function relationships have been obtained $\left(\mathrm{v}_{\mathrm{l}}, \mathrm{v}_{\mathrm{t}}\right.$ and $\mathrm{v}_{\mathrm{m}}$ were expressed in $\mathrm{km} / \mathrm{s}$, and $\mathrm{p}$ in $\left.\mathrm{GPa}\right)$ :

$$
\begin{aligned}
& \mathrm{V}_{\mathrm{l}}=7.97-0.57 \mathrm{p}+8.02 \times 10^{-2} \mathrm{p}^{2}-4.06 \times 10^{-3} \mathrm{p}^{3} \\
& \mathrm{~V}_{\mathrm{t}}=4.44-0.36 \mathrm{p}+4.60 \times 10^{-2} \mathrm{p}^{2}-2.31 \times 10^{-3} \mathrm{p}^{3} \\
& \mathrm{~V}_{\mathrm{m}}=4.95-0.40 \mathrm{p}+5.10 \times 10^{-2} \mathrm{p}^{2}-2.56 \times 10^{-3} \mathrm{p}^{3}
\end{aligned}
$$

There are various approximations to esteem the Grüneisen parameter $\gamma$, such as the thermodynamic $\gamma$, mode-specific $\gamma$, and acoustic $\gamma$ [13]. This later quantity $(\gamma)$ depends on the longitudinal $\left(\mathrm{v}_{\mathrm{l}}\right)$ and transverse $\left(\mathrm{v}_{\mathrm{t}}\right)$ sound velocities, it can be estimated by the following expression [12], [13]

$$
\gamma=(9 / 2)\left[v_{l}^{2}-(4 / 3) v_{t}^{2}\right] /\left[v_{l}^{2}+2 v_{t}^{2}\right]
$$

At $\mathrm{P}=0$, the value of the Grüneisen parameter $\gamma$ has been calculated and found to be 1.63 ; our obtained value of $\gamma$ is slightly bigger than the value 1.49 reported by Adachi [10].

\subsection{Surface acoustic wave speeds}

The speed of the sound plays a very important role in the surface acoustic wave (SAW) devices, filters, signal processing units, sensors and actuators [14], and widely used in different fields. Besides, the study of the elastic wave velocities for some specific crystallographic directions, the surface acoustic wave speeds of the AlP compound under high pressure have been calculated for the first time. The speed of the surface wave's Vsw in a B3 crystal propagating in the [100] and [110] directions are given as [15], [16]:

$$
\begin{aligned}
& C_{11}\left(V_{s w}^{2}-\frac{C_{44}}{g}\right)\left(V_{s w}^{2}-\frac{C_{11}}{g}+\frac{C_{12}^{2}}{C_{11} g}\right)^{2}=C_{44} V_{s w}^{4}\left(V_{s w}^{2}-\frac{C_{11}}{g}\right) \\
& C_{11}\left(V_{s w}^{2}-\frac{C_{44}}{g}\right)\left(V_{s w}^{2}-R+\frac{C_{12}^{2}}{C_{11} g}\right)^{2}=C_{44} V_{s w}^{4}\left(V_{s w}^{2}-R\right)
\end{aligned}
$$

Where: $\mathrm{R}$ is expressed as follow: $\mathrm{R}=\left(\left(\mathrm{C}_{11}+\mathrm{C}_{12}+2 \mathrm{C}_{44}\right) / 2 \mathrm{~g}\right)$. Equations (6) and (7) are of third degree in $\mathrm{V}^{2}$ sw refer to the [100] and [110] directions, respectively. The pressure dependence of the surface acoustic wave speeds $\left(\mathrm{V}_{\mathrm{sw}}\right)$ propagating in the [100] and [110] directions is plotted in Figure 2.



Fig. 2: Surface-Acoustic Wave Speeds Propagating in the [100] and [110] Directions for AlP Material Versus Pressure.

It is observed that the surface acoustic wave speeds decreases with increasing pressure for both [100] and [110] directions. The solid lines in the curves represent the best fit of the calculated data and which can be approximated by the following analytical expressions respectively:

$$
\begin{aligned}
& \mathrm{V}_{\mathrm{sw}}\left(\mathrm{kms}^{-1}\right)=4.088-0.329 \mathrm{p}+4.17 \times 10^{-2} \mathrm{p}^{2}-2.07 \times 10^{-3} \mathrm{p}^{3} \\
& \mathrm{~V}_{\mathrm{sw}}\left(\mathrm{kms}^{-1}\right)=4.335-0.343 \mathrm{p}+4.45 \times 10^{-2} \mathrm{p}^{2}-2.25 \times 10^{-3} \mathrm{p}^{3}
\end{aligned}
$$

It is important to note that the same behavior of the surface acoustic wave speeds under compression was also observed in cubic zincblende AlN compound [16] for both [100] and [110] directions. To the best of authors' knowledge, there are no data available in the literature on the surface acoustic wave speeds for AlP semiconducting material.

\subsection{Vickers and knoop microhardness}

The mechanical behavior of solid can be specified by means of the microhardness, which can be calculated by various methods [15]. For the semiconductors, the Knoop and the Vickers indentations are usually used. The Vickers hardness Hv can be calculated by the following formula [1]: 
$H_{v}=2\left(G^{3} / B^{2}\right)^{0.585}-3$

Where $\mathrm{G}$ is the shear modulus, and $\mathrm{B}$ is the bulk modulus.

For some cubic semiconductors, the Knoop microhardness H and the Voigt shear modulus where Gv are related by [10]:

$\mathrm{H}=0.139 \mathrm{Gv}$

Our values of the Vickers hardness (Hv) and the Knoop microhardness H were found to be 6.11 and $6.96 \mathrm{GPa}$, respectively. The calculated values are respectively slightly smaller than the theoretical one (7.0 GPa) reported in Ref. [1], and higher than the experimental one $(5.5 \mathrm{GPa})$ quoted by Adachi [10]. For some other cubic materials of group-IV, III-V and II-VI semiconductors, Adachi [10] proposed a relationship between the Knoop microhardness $\mathrm{H}$ and the inverse unit-cell volume $\left(\mathrm{V}^{-1}=\mathrm{a}^{-3}\right)$, and expressed as follow: $\mathrm{H}=(3220 / \mathrm{V})$ 14.6, where: $\mathrm{V}$ is in $\AA^{3}$ and $\mathrm{H}$ is in GPa. Using the value of the lattice constant $(\mathrm{a}=5.436 \AA$ ) [7], the Knoop microhardness $\mathrm{H}$ of AlP was found to be $5.45 \mathrm{GPa}$; which is in excellent agreement with the experimental one (5.5 GPa) quoted by Adachi [10], the deviation between the two values is only about $0.91 \%$.

\section{Conclusion}

Using the elastic constants and structural parameters obtained in our previous work published in J. Optoelectron. Adv. M. 16, 207 (2014), the bulk and surface acoustic wave velocities of Aluminum phosphide (AlP) under pressure up to 9.5 GPa were studied. The obtained results at zero-pressure are analyzed and compared with previous data of the literature. In general our findings are in good agreement with other data of the literature.

In addition, the Grüneisen parameter and the Vickers and Knoop microhardness are predicted and analyzed in details. Our obtained results of the microhardness are also in agreement with other data of the literature.

\section{References}

[1] R. Yang, C. Zhu, Q. Wei, and D. Zhang, "First-principles study on phases of AlP ", Solid State Communications, Vol. 267, (2017), pp. 23-28. https://doi.org/10.1016/j.ssc.2017.09.008.

[2] S. Aouadi, P. Rodriguez-Hernandez, K. Kassali, and A. Muñoz, " Lattice dynamics properties of zinc-blende and nickel arsenide phases of AlP ", Physics Letters A, Vol. 372, No. 32, (2008), pp. 5340 -5345. https://doi.org/10.1016/j.physleta.2008.06.010.

[3] S. Lakel, F. Okbi, M. Ibrir, and K. Almi, "Pressure effects on the elastic and lattice dynamics properties of AlP from first principles calculations", AIP Conference Proceedings. Vol. 1653, No. 1, (2015), pp. 020065 (7 pages). https://doi.org/10.1063/1.4914256.

[4] M. Ameri, A. Bentouaf, M. D. Aici, R. Khenata, F. Boufadi, and A. Touia, "Structural and Electronic Properties Calculations of Al $\mathrm{In}_{1-\mathrm{x}} \mathrm{P} \mathrm{Alloy}$, Materials Sciences and Application, Vol. 2, No. 32, (2011), pp. 729-738. https://doi.org/10.4236/msa.2011.27101.

[5] A. Bouhemadou, R. Khenata, M. Kharoubi, T. Seddik, Ali H. Reshak, and Y. Al-Douri, "FP-APW + lo calculations of the elastic properties in zinc-blende III-P compounds under pressure effects", Computational Materials Science. Vol. 45, No. 2, (2009), pp. 474-479. https://doi.org/10.1016/j.commatsci.2008.11.013.

[6] S. Ehsanfar, F. Kanjouri, H. Tashakori, and A. Esmailian, " First-principles study of structural, electronic, mechanical, thermal, and phonon properties of III-Phosphides (BP, AlP, GaP, and InP) ", Journal of Electronic Materials, Vol. 46, No. 10, (2017), pp. 6214-6223. https://doi.org/10.1007/s11664-017-5640-4.

[7] S. Daoud, N. Bioud, and N. Lebga, " Structural, elastic, piezoelectric and electronic properties of (B3) AlP compound under pressure ", Journal of Optoelectronics and Advanced Materials, Vol. 16, No. 1-2, (2014), pp. 207 - 214.

[8] D. Singh, G. Mishra, R. Kumar, and R.R. Yadav, "Temperature dependence of elastic and ultrasonic properties of sodium borohydride ", Communications in Physics, Vol. 27, No. 2, (2017), pp. 151-164. https://doi.org/10.15625/0868-3166/27/2/9615.

[9] H. Ma, J. Zhang, B. Zhao, Q. Wei, and Y. Yang, "First-principles study on mechanical and elastic properties of $\mathrm{B}_{\mathrm{x}} \mathrm{Al}_{1-\mathrm{X}} \mathrm{P}$ alloys", AIP Advances Vol. 7, No. 6, (2017), pp. 065007 (12 pages). https://doi.org/10.1063/1.4985254.

[10] S. Adachi, Properties of Group-IV, III-V and II-VI Semiconductors, John Wiley \& Sons, England, (2005). ISBN 0-470-09032-4. https://doi.org/10.1002/0470090340.

[11] N. Lebga, S. Daoud, X-W. Sun, N. Bioud, and A. Latreche, "Mechanical and thermophysical properties of cubic rock-salt AlN under high pressure", Journal of Electronic Materials, Vol. 47, No. 7, (2018), pp. 3430-3439. https://doi.org/10.1007/s11664-018-6169-x.

[12] S. Daoud, "Sound velocities and thermal properties of BeX (X=S, Se and Te) alkaline-earth chalcogenides", International Journal of Scientific World, Vol. 5, No.1, (2017), pp. 9-12. https://doi.org/10.14419/ijsw.v5i1.6929.

[13] Z. Tian, L. Sun, J. Wang, and J. Wang, "Theoretical prediction and experimental determination of the low lattice thermal conductivity of $\mathrm{Lu}_{2} \mathrm{SiO}_{5}$ ", Journal of the European Ceramic Society Vol. 35, No. 6, (2015), pp. 1923-1932. https://doi.org/10.1016/j.jeurceramsoc.2015.01.001.

[14] B. Liu, X. Chen, H. Cai, M. Mohammad Ali, X. Tian, X., L.Tao, Y.Yang, and T. Ren, "Surface acoustic wave devices for sensor applications", Journal of Semiconductors, Vol. 37, No. 2, (2016), pp. 021001 (9 pages). https://doi.org/10.1088/1674-4926/37/2/021001.

[15] N. Bouarissa, and Y. Atik, " Elastic constants and acoustic wave velocities in $\mathrm{Cd}_{1-x} \mathrm{Zn}_{\mathrm{x}} \mathrm{Te}$ mixed crystals", Modern Physics Letters B, Vol. 22, No. 12, (2008), pp. 1221-1229. https://doi.org/10.1142/S0217984908015371.

[16] S. Daoud, and N. Bouarissa, "Elastic, piezoelectric and thermal properties of zinc-blende AlN under pressure ", Theoretical Chemistry Accounts, Vol. 138, No. 4, (2019), pp. 49 (10 pages). https://doi.org/10.1007/s00214-019-2439-9. 